\title{
ANALYTICAL ASPECTS OF HEAT FLOW IN RANDOM TWO-COMPONENT LAMINATES*
}

\author{
BY \\ J. M. HILL ${ }^{1}$ AND J. R. WILLIS \\ University of Bath, Claverton Down, Bath, England
}

\begin{abstract}
Analytical aspects are examined for the coupled reaction-diffusion equations arising for the expected conditional temperatures for heat flow in a random two-component laminate with heat flowing in a direction perpendicular to the laminae. The basic equations derived by Clarke [1] involve new terms not previously encountered within the contexts of either diffusion or heat flows. Various results and solution procedures are developed for both the coupled system and the underlying fourth-order equation. Basic source solutions for the coupled system are derived which are extended to general solutions of the coupled system with the new terms. These general solutions involve two arbitrary heat functions and display explicitly the dependence of solutions on the various parameters of the model. However, these general solutions appear not to be as useful in the context of the solution of boundary value problems as the corresponding results for the coupled system without the new terms. A simple illustrative example is provided for the utilization of such general solutions for a specific problem.
\end{abstract}

1. Introduction. In a recent paper, Clarke [1] obtains the following coupled reaction-diffusion equations for variables $T_{j}(x, t)(j=1,2)$, which represent expectation values of temperature at position $x$ and time $t$, conditional upon material $j$ being present at $x$, in a random two-component laminate, for heat flow in a direction perpendicular to the laminae:

$$
\begin{aligned}
& \nu_{1} \frac{\partial T_{1}}{\partial t}=\lambda_{1} \frac{\partial^{2} T_{1}}{\partial x^{2}}+\beta p_{2}\left(\lambda_{1}-\lambda_{2}\right) \frac{\partial T_{2}}{\partial x}-\beta^{2} p_{2}\left(p_{1} \lambda_{2}+p_{2} \lambda_{1}\right)\left(T_{1}-T_{2}\right), \\
& \nu_{2} \frac{\partial T_{2}}{\partial t}=\lambda_{2} \frac{\partial^{2} T_{2}}{\partial x^{2}}+\beta p_{1}\left(\lambda_{2}-\lambda_{1}\right) \frac{\partial T_{1}}{\partial x}-\beta^{2} p_{1}\left(p_{1} \lambda_{2}+p_{2} \lambda_{1}\right)\left(T_{2}-T_{1}\right) .
\end{aligned}
$$

In these equations, $\nu_{j}$ and $\lambda_{j}(j=1,2)$ denote the constant heat capacities and thermal conductivities respectively. Further, $p_{1}$ and $p_{2}$ are the volume fractions of the two components so that $p_{1}+p_{2}=1$ and $\beta$ is a constant characterizing the construction of the random medium. Clarke assumes that a realization of the medium

*Received May 12, 1987.

Permanent address: Department of Mathematics, University of Wollongong, N.S.W., AUSTRALIA. 
is generated from a generalized random telegraph process; the constant $\beta$ is a measure of the number of material changes per unit distance or, equivalently, $\beta^{-1}$ is a measure of the average distance between changes. Such issues are discussed in detail in [1] where, in addition, the basic equations for an $N$-component laminate may also be found for the cases of heat flow both parallel and perpendicular to the laminae.

In a subsequent paper, for a slightly more general random process, Clarke [2] derives the basic equations for inclined random laminates which incorporate the situations of heat flow both along and across the laminae as special cases. Here we confine our attention only to equations of the type (1.1) since the essential new mathematical feature of Clarke's model is the appearance of the middle terms on the right-hand sides of equations (1.1). We emphasize that these terms are of course not the usual convection terms arising in heat and diffusion phenomena and, as far as the authors are aware, they do not appear to have arisen previously in the contexts of either diffusion or heat conduction. Further we note that, for heat flow along the laminae, Clarke [1] obtains precisely (1.1) but without the new terms so that these terms appear to reflect the continual changes of the media. Accordingly, we adopt the following as our basic system of equations:

$$
\begin{aligned}
& \frac{\partial T_{1}}{\partial t}=D_{1} \frac{\partial^{2} T_{1}}{\partial x^{2}}+e_{1} \frac{\partial T_{2}}{\partial x}-f_{1}\left(T_{1}-T_{2}\right), \\
& \frac{\partial T_{2}}{\partial t}=D_{2} \frac{\partial^{2} T_{2}}{\partial x^{2}}+e_{2} \frac{\partial T_{1}}{\partial x}-f_{2}\left(T_{2}-T_{1}\right),
\end{aligned}
$$

where the six real constants $D_{j}, e_{j}$, and $f_{j}(j=1,2)$ are given by

$$
\begin{array}{lll}
D_{1}=\frac{\lambda_{1}}{\nu_{1}}, & e_{1}=\beta \frac{p_{2}}{\nu_{1}}\left(\lambda_{1}-\lambda_{2}\right), & f_{1}=\beta^{2} \frac{p_{2}}{\nu_{1}}\left(p_{1} \lambda_{2}+p_{2} \lambda_{1}\right), \\
D_{2}=\frac{\lambda_{2}}{\nu_{2}}, & e_{2}=\beta \frac{p_{1}}{\nu_{2}}\left(\lambda_{2}-\lambda_{1}\right), & f_{2}=\beta^{2} \frac{p_{1}}{\nu_{2}}\left(p_{1} \lambda_{2}+p_{2} \lambda_{1}\right),
\end{array}
$$

and the following important relations may be readily verified:

$$
\beta^{2}=\frac{f_{1}}{D_{1}}+\frac{f_{2}}{D_{2}}+\frac{e_{1} e_{2}}{D_{1} D_{2}}, \quad e_{1} f_{2}+e_{2} f_{1}=0 .
$$

The purpose of this paper is to describe various results for (1.2) relating to solutions and solution procedures for this system. In the following two sections we show how we may utilize known results for the special cases of either equal thermal conductivities or equal heat capacities. In the section thereafter we examine the fourth-order equation arising from (1.2) which both $T_{1}(x, t)$ and $T_{2}(x, t)$ satisfy. The unconditional expected temperature $T(x, t)$, given by

$$
T(x, t)=p_{1} T_{1}(x, t)+p_{2} T_{2}(x, t),
$$

also satisfies this equation. In the final sections of the paper we deal with general solutions of (1.2) without additional assumptions on the thermal conductivities or heat capacities. The general solutions of (1.2) given in Sec. 6 originate from the basic source solutions of (1.2) which we derive in Sec. 5. Finally, in Sec. 7, we illustrate the solution procedure for a specific simple problem. We emphasize that in this paper we deal exclusively with mathematical aspects of (1.2). In a related paper, 
Willis and Hill [6], we re-examine the basic foundations of heat diffusion in random laminates and we propose a possible alternative formulation.

2. Equal thermal conductivities. For the special case of equal thermal conductivities $\lambda_{1}=\lambda_{2}$ (or for heat flow along the laminae) we have a system of equations of the form

$$
\frac{\partial T_{1}}{\partial t}=D_{1} \frac{\partial^{2} T_{1}}{\partial x^{2}}-f_{1}\left(T_{1}-T_{2}\right), \quad \frac{\partial T_{2}}{\partial t}=D_{2} \frac{\partial^{2} T_{2}}{\partial x^{2}}-f_{2}\left(T_{2}-T_{1}\right) .
$$

Formal solutions of (2.1) may be found in Hill [3] as

$$
\begin{aligned}
T_{1}(x, t)= & e^{-f_{1} t} h_{1}\left(x, D_{1} t\right) \\
& +\frac{f_{1}^{1 / 2} e^{\delta t}}{\left(D_{1}-D_{2}\right)} \int_{D_{2} t}^{D_{1} t} e^{-\gamma \xi}\left\{f_{2}^{1 / 2}\left(\frac{\xi-D_{2} t}{D_{1} t-\xi}\right)^{1 / 2} I_{1}(\eta) h_{1}(x, \xi)\right. \\
& \left.+f_{1}^{1 / 2} I_{0}(\eta) h_{2}(x, \xi)\right\} d \xi, \\
T_{2}(x, t)= & e^{-f_{2} t} h_{2}\left(x, D_{2} t\right) \\
& +\frac{f_{2}^{1 / 2} e^{\delta t}}{\left(D_{1}-D_{2}\right)} \int_{D_{2} t}^{D_{1} t} e^{-\gamma \xi}\left\{f_{1}^{1 / 2}\left(\frac{D_{1} t-\xi}{\xi-D_{2} t}\right)^{1 / 2} I_{1}(\eta) h_{2}(x, \xi)\right. \\
& \left.+f_{2}^{1 / 2} I_{0}(\eta) h_{1}(x, \xi)\right\} d \xi,
\end{aligned}
$$

where the constants $\gamma$ and $\delta$ are defined by

$$
\gamma=\left(\frac{f_{1}-f_{2}}{D_{1}-D_{2}}\right), \quad \delta=\left(\frac{f_{1} D_{2}-f_{2} D_{1}}{D_{1}-D_{2}}\right),
$$

and $I_{0}$ and $I_{1}$ are the usual modified Bessel functions. Further, $\eta$ is given by

$$
\eta=\frac{2\left(f_{1} f_{2}\right)^{1 / 2}}{\left(D_{1}-D_{2}\right)}\left[\left(D_{1} t-\xi\right)\left(\xi-D_{2} t\right)\right]^{1 / 2}
$$

and $h_{1}(x, t)$ and $h_{2}(x, t)$ are solutions of the classical heat equation

$$
\frac{\partial h_{j}}{\partial t}=\frac{\partial^{2} h_{j}}{\partial x^{2}} \quad(j=1,2)
$$

satisfying the same initial data as $T_{1}(x, t)$ and $T_{2}(x, t)$, respectively, and it is assumed that $D_{1}>D_{2}$.

The main merits of these representations are, firstly, the given explicit dependence of the solutions on the four parameters $D_{j}$ and $f_{j}(j=1,2)$ and, secondly, the possibility of reducing a boundary value problem for the coupled system to two boundary value problems for the classical heat equation. Related issues and examples may be found in [3]. Here we simply remark that for coupled reaction-diffusion systems, solutions such as (2.2) are important general results for the system and, accordingly, the question naturally arises as to the appropriate extension of (2.2) which applies to the coupled system (1.2). The appropriate generalization is by no means obvious since, in general, (1.2) cannot be reduced to a system of the form (2.1) by simple transformations. In order to obtain the general solutions applying to 
(1.2) we must follow the procedure for the derivation of (2.2), that is, we must first establish the source solutions for (1.2), which is done in Sec. 5. However, in the following two sections, we first develop more immediate results, which follow from (1.2) by known results and more elementary devices.

3. Flux equations and equal heat capacities. Clarke [1] deduces (1.1) by eliminating the expected conditional fluxes $Q_{j}(x, t)(j=1,2)$ from

$$
\frac{Q_{j}}{\lambda_{j}}=-\frac{\partial T_{j}}{\partial x}-\beta\left(T-T_{j}\right), \quad \nu_{j} \frac{\partial T_{j}}{\partial t}+\frac{\partial Q_{j}}{\partial x}=\beta\left(Q-Q_{j}\right),
$$

where the unconditional expected temperature $T(x, t)$ and flux $Q(x, t)$ are given, respectively, by (1.5) and

$$
Q(x, t)=p_{1} Q_{1}(x, t)+p_{2} Q_{2}(x, t) .
$$

Thus we have

$$
\begin{array}{cl}
\frac{Q_{1}}{\lambda_{1}}=-\frac{\partial T_{1}}{\partial x}+\beta p_{2}\left(T_{1}-T_{2}\right), & \frac{Q_{2}}{\lambda_{2}}=-\frac{\partial T_{2}}{\partial x}+\beta p_{1}\left(T_{2}-T_{1}\right), \\
\nu_{1} \frac{\partial T_{1}}{\partial t}+\frac{\partial Q_{1}}{\partial x}=-\beta p_{2}\left(Q_{1}-Q_{2}\right), & \nu_{2} \frac{\partial T_{2}}{\partial t}+\frac{\partial Q_{2}}{\partial x}=-\beta p_{1}\left(Q_{2}-Q_{1}\right),
\end{array}
$$

and it may be readily verified that (1.1) emerges on eliminating $Q_{j}(x, t)(j=1,2)$. Conversely, on eliminating $T_{j}(x, t)(j=1,2)$ we obtain

$$
\begin{aligned}
& \frac{1}{\lambda_{1}} \frac{\partial Q_{1}}{\partial t}=\frac{1}{\nu_{1}} \frac{\partial^{2} Q_{1}}{\partial x^{2}}+\beta p_{2}\left(\frac{1}{\nu_{2}}-\frac{1}{\nu_{1}}\right) \frac{\partial Q_{2}}{\partial x}-\beta^{2} p_{2}\left(\frac{p_{1}}{\nu_{2}}+\frac{p_{2}}{\nu_{1}}\right)\left(Q_{1}-Q_{2}\right), \\
& \frac{1}{\lambda_{2}} \frac{\partial Q_{2}}{\partial t}=\frac{1}{\nu_{2}} \frac{\partial^{2} Q_{2}}{\partial x^{2}}+\beta p_{1}\left(\frac{1}{\nu_{1}}-\frac{1}{\nu_{2}}\right) \frac{\partial Q_{1}}{\partial x}-\beta^{2} p_{1}\left(\frac{p_{1}}{\nu_{2}}+\frac{p_{2}}{\nu_{1}}\right)\left(Q_{2}-Q_{1}\right) .
\end{aligned}
$$

Thus we again have a system of the form (1.2), namely

$$
\begin{aligned}
& \frac{\partial Q_{1}}{\partial t}=D_{1} \frac{\partial^{2} Q_{1}}{\partial x^{2}}+E_{1} \frac{\partial Q_{2}}{\partial x}-F_{1}\left(Q_{1}-Q_{2}\right), \\
& \frac{\partial Q_{2}}{\partial t}=D_{2} \frac{\partial^{2} Q_{2}}{\partial x^{2}}+E_{2} \frac{\partial Q_{1}}{\partial x}-F_{2}\left(Q_{2}-Q_{1}\right),
\end{aligned}
$$

where the six real constants $D_{j}, E_{j}$, and $F_{j}(j=1,2)$ are given by

$$
\begin{array}{lll}
D_{1}=\frac{\lambda_{1}}{\nu_{1}}, & E_{1}=\beta p_{2} \lambda_{1}\left(\frac{1}{\nu_{2}}-\frac{1}{\nu_{1}}\right), & F_{1}=\beta^{2} p_{2} \lambda_{1}\left(\frac{p_{1}}{\nu_{2}}+\frac{p_{2}}{\nu_{1}}\right), \\
D_{2}=\frac{\lambda_{2}}{\nu_{2}}, & E_{2}=\beta p_{1} \lambda_{2}\left(\frac{1}{\nu_{1}}-\frac{1}{\nu_{2}}\right), & F_{2}=\beta^{2} p_{1} \lambda_{2}\left(\frac{p_{1}}{\nu_{2}}+\frac{p_{2}}{\nu_{1}}\right),
\end{array}
$$

which evidently satisfy relations similar to (1.4).

It is apparent from (3.4) that the special case of equal heat capacities, $\nu_{1}=\nu_{2}$, admits general solutions of the form (2.2) with $F_{1}$ and $F_{2}$ in place of $f_{1}$ and $f_{2}$ respectively. Assuming, therefore, that $Q_{j}(x, t)(j=1,2)$ are known functions we may deduce expressions for $T_{j}(x, t)(j=1,2)$ by integration of $(3.3)_{1}$ and $(3.3)_{2}$. 
Thus, for example, for the semi-infinite medium occupying $0<x<\infty$ with zero temperature at infinity we may readily deduce

$$
\begin{aligned}
& T_{1}(x, t)=\int_{x}^{\infty}\left\{\left[p_{1}+p_{2} e^{\beta(x-X)}\right] \frac{Q_{1}(X, t)}{\lambda_{1}}+p_{2}\left[1-e^{\beta(x-X)}\right] \frac{Q_{2}(X, t)}{\lambda_{2}}\right\} d X, \\
& T_{2}(x, t)=\int_{x}^{\infty}\left\{p_{1}\left[1-e^{\beta(x-X)}\right] \frac{Q_{1}(X, t)}{\lambda_{1}}+\left[p_{2}+p_{1} e^{\beta(x-X)}\right] \frac{Q_{2}(X, t)}{\lambda_{2}}\right\} d X,
\end{aligned}
$$

so that, with $Q_{j}(x, t)(j=1,2)$ given by expressions of the form (2.2), equation (3.7) constitutes the temperature solution. In the following section we analyze the fourth-order partial differential equation arising from (1.2).

4. Fourth-order equation. On writing the coupled system (1.2) as

$$
\left[\begin{array}{ll}
\frac{\partial}{\partial t}-D_{1} \frac{\partial^{2}}{\partial x^{2}}+f_{1} & -\left(e_{1} \frac{\partial}{\partial x}+f_{1}\right) \\
-\left(e_{2} \frac{\partial}{\partial x}+f_{2}\right) & \frac{\partial}{\partial t}-D_{2} \frac{\partial}{\partial x^{2}}+f_{2}
\end{array}\right]\left(\begin{array}{l}
T_{1} \\
T_{2}
\end{array}\right)=\left(\begin{array}{l}
0 \\
0
\end{array}\right)
$$

the fourth-order equation satisfied by $T_{1}(x, t)$ and $T_{2}(x, t)$, and therefore the unconditional expected temperature $T(x, t)$ given by $(1.5)$, is obtained by equating the determinant to zero. On using the relations (1.4) this equation becomes

$$
\frac{\partial^{2} T}{\partial t^{2}}+\left(f_{1}+f_{2}\right) \frac{\partial T}{\partial t}=D_{1} D_{2}\left\{\beta^{2} \frac{\partial^{2} T}{\partial x^{2}}+\left(\frac{1}{D_{1}}+\frac{1}{D_{2}}\right) \frac{\partial^{3} T}{\partial x^{2} \partial t}-\frac{\partial^{4} T}{\partial x^{4}}\right\} .
$$

Now the large time thermal diffusivity $D^{*}$ arises simply by approximating (4.2) by

$$
\left(f_{1}+f_{2}\right) \frac{\partial T}{\partial t} \simeq D_{1} D_{2} \beta^{2} \frac{\partial^{2} T}{\partial x^{2}}
$$

so that

$$
D^{*}=\frac{D_{1} D_{2} \beta^{2}}{\left(f_{1}+f_{2}\right)}=\frac{1}{\left(p_{1} \nu_{1}+p_{2} \nu_{2}\right)\left(\frac{p_{1}}{\lambda_{1}}+\frac{p_{2}}{\lambda_{2}}\right)}
$$

By a similar argument the large-time thermal diffusivity $D^{\dagger}$ for heat flow parallel to the laminae is given by

$$
D^{\dagger}=\frac{\left(f_{1} D_{2}+f_{2} D_{1}\right)}{\left(f_{1}+f_{2}\right)}=\frac{\left(p_{1} \lambda_{1}+p_{2} \lambda_{2}\right)}{\left(p_{1} \nu_{1}+p_{2} \nu_{2}\right)}
$$

and we note that (4.4) and (4.5) are in complete agreement with Clarke [1] whose results were obtained from more elaborate considerations. We also observe that, while the coupled system (1.2) appears not to be invariant under transformations $x \leftrightarrow-x$, the fourth-order equation is indeed unchanged by this transformation.

The detailed solution of boundary value problems for equations of the form (4.2) is described in Lee and Hill [4]. Here we observe that, with the notation

$$
L_{j}=\frac{\partial}{\partial t}-D_{j} \frac{\partial^{2}}{\partial x^{2}} \quad(j=1,2),
$$

we obtain the following alternative form of (4.2) on equating the determinant in (4.1) to zero:

$$
\left\{L_{1} L_{2}+\left(f_{1} L_{2}+f_{2} L_{1}\right)-e_{1} e_{2} \frac{\partial^{2}}{\partial x^{2}}\right\} T=0
$$


On noting the relation

this simplifies to give

$$
\frac{\partial^{2}}{\partial x^{2}}=-\frac{1}{\left(D_{1}-D_{2}\right)}\left\{L_{1}-L_{2}\right\}
$$

$$
\left[L_{1} L_{2}+\left(\gamma_{1} L_{2}+\gamma_{2} L_{1}\right)\right] T=0
$$

where the constants $\gamma_{j}(j=1,2)$ are defined by

$$
\gamma_{1}=f_{1}-\frac{e_{1} e_{2}}{\left(D_{1}-D_{2}\right)}, \quad \gamma_{2}=f_{2}+\frac{e_{1} e_{2}}{\left(D_{1}-D_{2}\right)} .
$$

Thus, on introducing new operators $L_{j}^{*}(j=1,2)$ defined by

$$
L_{j}^{*}=L_{j}+\gamma_{j} \quad(j=1,2),
$$

we observe that (4.9) can be written as

$$
\left(L_{1}^{*} L_{2}^{*}-\gamma_{1} \gamma_{2}\right) T=0,
$$

and, in particular, any solutions of the coupled system

$$
L_{1}^{*} u_{1}=\gamma_{1} u_{2}, \quad L_{2}^{*} u_{2}=\gamma_{2} u_{1},
$$

are also solutions of (4.12). Writing these equations in full gives

$$
\frac{\partial u_{1}}{\partial t}=D_{1} \frac{\partial^{2} u_{1}}{\partial x^{2}}-\gamma_{1}\left(u_{1}-u_{2}\right), \quad \frac{\partial u_{2}}{\partial t}=D_{2} \frac{\partial^{2} u_{2}}{\partial x^{2}}-\gamma_{2}\left(u_{2}-u_{1}\right),
$$

which accordingly have solutions of the form (2.2) with $f_{1}$ and $f_{2}$ replaced by $\gamma_{1}$ and $\gamma_{2}$ respectively.

In summary, the above analysis demonstrates that the fourth-order equation (4.2) admits solutions of the form (2.2) with constants $\gamma_{j}$ in place of $f_{j}(j=1,2)$ so that the appropriate $\gamma$ and $\delta$ are given by

$$
\begin{aligned}
& \gamma=\left(\frac{\gamma_{1}-\gamma_{2}}{D_{1}-D_{2}}\right)=\left(\frac{f_{1}-f_{2}}{D_{1}-D_{2}}\right)-\frac{2 e_{1} e_{2}}{\left(D_{1}-D_{2}\right)^{2}}, \\
& \delta=\left(\frac{\gamma_{1} D_{2}-\gamma_{2} D_{1}}{D_{1}-D_{2}}\right)=\left(\frac{f_{1} D_{2}-f_{2} D_{1}}{D_{1}-D_{2}}\right)-\frac{e_{1} e_{2}\left(D_{1}+D_{2}\right)}{\left(D_{1}-D_{2}\right)^{2}},
\end{aligned}
$$

with $\eta$ defined by

$$
\eta=\frac{2 k^{1 / 2}}{\left(D_{1}-D_{2}\right)}\left[\left(D_{1} t-\xi\right)\left(\xi-D_{2} t\right)\right]^{1 / 2},
$$

where the constant $k$ is given by

$$
k=\gamma_{1} \gamma_{2}=f_{1} f_{2}+\left(\frac{f_{1}-f_{2}}{D_{1}-D_{2}}\right) e_{1} e_{2}-\left(\frac{e_{1} e_{2}}{D_{1}-D_{2}}\right)^{2} .
$$

However, the resulting solutions of (4.14) are evidently not also solutions of the original coupled system (1.2) and the importance of the preceding analysis is the identification of the above constants $\gamma, \delta$, and $k$ and their dependence on the six constants $D_{j}, e_{j}$, and $f_{j}(j=1,2)$ of the given system. In the following two sections we demonstrate how genuine solution pairs $\left\{T_{1}, T_{2}\right\}$ of the original coupled system (1.2) may be generated and how $\gamma_{1}, \gamma_{2}, \gamma, \delta$, and $k$ emerge as fundamental constants of the system. 
Finally, in this section we make some additional observations relating to the fourthorder equation and the large-time behaviour. Firstly, (4.2) admits the alternative form

$$
\begin{aligned}
\left(\frac{\partial}{\partial t}-D_{1} \frac{\partial^{2}}{\partial x^{2}}\right)\left(\frac{\partial}{\partial t}\right. & \left.-D_{2} \frac{\partial^{2}}{\partial x^{2}}\right) T \\
+ & \beta^{2}\left(\frac{p_{1}}{\nu_{2}}+\frac{p_{2}}{\nu_{1}}\right)\left(p_{1} \lambda_{2}+p_{2} \lambda_{1}\right)\left(\frac{\partial}{\partial t}-D^{*} \frac{\partial^{2}}{\partial x^{2}}\right) T=0,
\end{aligned}
$$

where $D^{*}$ is the large-time diffusivity given by (4.4). Roughly speaking, we may interpret the terms of this equation as small-time and large-time contributions in the sense that the first term reflects the fact that for small times the coupling terms in (3.3) are not significant and the short-time diffusivities are simply $D_{1}$ and $D_{2}$, while for large times the second term of (4.18) dominates and the temperature satisfies the classical equation with diffusivity $D^{*}$. Secondly, for large times the following alternative derivation of the classical equation may be of interest. From (3.3) we may readily deduce the overall relations

$$
p_{1} \frac{Q_{1}}{\lambda_{1}}+p_{2} \frac{Q_{2}}{\lambda_{2}}=-\frac{\partial T}{\partial x}, \quad p_{1} \nu_{1} \frac{\partial T_{1}}{\partial t}+p_{2} \nu_{2} \frac{\partial T_{2}}{\partial t}+\frac{\partial Q}{\partial x}=0,
$$

so that for large times, since $T_{1} \simeq T_{2} \simeq T$ and $Q_{1} \simeq Q_{2} \simeq Q$, we have

$$
Q \simeq-\frac{1}{\left(\frac{p_{1}}{\lambda_{1}}+\frac{p_{2}}{\lambda_{2}}\right)} \frac{\partial T}{\partial x}, \quad\left(p_{1} \nu_{1}+p_{2} \nu_{2}\right) \frac{\partial T}{\partial t}+\frac{\partial Q}{\partial x} \simeq 0,
$$

from which the classical equation with diffusivity $D^{*}$ follows immediately.

5. Source solutions. In order to deduce general solutions of (1.2) we first need to identify the structure of the source solutions for $-\infty<x<\infty$, that is, solutions of (1.2) vanishing at infinity such that, initially,

$$
T_{1}(x, 0)=\rho_{1} \delta(x), \quad T_{2}(x, 0)=\rho_{2} \delta(x),
$$

where $\rho_{j}(j=1,2)$ denote the constant source strengths and $\delta(x)$ is the usual Dirac delta function. On applying the Fourier transform

$$
\bar{T}_{j}(\omega, t)=\int_{-\infty}^{\infty} e^{-i \omega x} T_{j}(x, t) d x=2 \int_{0}^{\infty} \cos \omega x T_{j}(x, t) d x,
$$

and then the Laplace transform

$$
\overline{\bar{T}}_{j}(\omega, s)=\int_{0}^{\infty} e^{-s t} \bar{T}_{j}(\omega, t) d t,
$$

we may establish in a routine manner,

$$
\left[\begin{array}{cc}
D_{1} \omega^{2}+f_{1}+s & -\left(f_{1}+i \omega e_{1}\right) \\
-\left(f_{2}+i \omega e_{2}\right) & D_{2} \omega^{2}+f_{2}+s
\end{array}\right]\left(\begin{array}{c}
\overline{\bar{T}}_{1} \\
\overline{\bar{T}}_{2}
\end{array}\right)=\left(\begin{array}{c}
\rho_{1} \\
\rho_{2}
\end{array}\right) .
$$

The determinant $\Delta$ of this matrix is given by

$$
\Delta=s^{2}+\left[\left(D_{1}+D_{2}\right) \omega^{2}+\left(f_{1}+f_{2}\right)\right] s+D_{1} D_{2} \omega^{2}\left(\omega^{2}+\beta^{2}\right),
$$


and it is not difficult to show that the roots $s_{1}(\omega)$ and $s_{2}(\omega)$ of $\Delta=0$ are given by

$$
s_{1}(\omega)=-\lambda+\left(\mu^{2}+k\right)^{1 / 2}, \quad s_{2}(\omega)=-\lambda-\left(\mu^{2}+k\right)^{1 / 2},
$$

where $\lambda$ and $\mu$ are functions of $\omega$ given by

$$
\lambda(\omega)=\frac{1}{2}\left\{\left(D_{1}+D_{2}\right) \omega^{2}+\left(\gamma_{1}+\gamma_{2}\right)\right\}, \quad \mu(\omega)=\frac{1}{2}\left\{\left(D_{1}-D_{2}\right) \omega^{2}+\left(\gamma_{1}-\gamma_{2}\right)\right\},
$$

and $\gamma_{1}, \gamma_{2}$, and $k$ are all as previously defined by (4.10) and (4.17).

Now from the solution of $(5.4)$,

$$
\begin{aligned}
& \overline{\bar{T}}_{1}(\omega, s)=\frac{\left(D_{2} \omega^{2}+f_{2}+s\right) \rho_{1}+\left(f_{1}+i \omega e_{1}\right) \rho_{2}}{\left(s-s_{1}\right)\left(s-s_{2}\right)}, \\
& \overline{\bar{T}}_{2}(\omega, s)=\frac{\left(f_{2}+i \omega e_{2}\right) \rho_{1}+\left(D_{1} \omega^{2}+f_{1}+s\right) \rho_{2}}{\left(s-s_{1}\right)\left(s-s_{2}\right)} .
\end{aligned}
$$

It is a simple matter to show that, after a Laplace inversion,

$$
\begin{aligned}
& \bar{T}_{1}(\omega, t)=\rho_{1} \frac{\partial \bar{p}}{\partial t}+\left[f_{2} \rho_{1}+\left(f_{1}+i \omega e_{1}\right) \rho_{2}\right] \bar{p}+D_{2} \rho_{1} \omega^{2} \bar{p}, \\
& \bar{T}_{2}(\omega, t)=\rho_{2} \frac{\partial \bar{p}}{\partial t}+\left[f_{1} \rho_{2}+\left(f_{2}+i \omega e_{2}\right) \rho_{1}\right] \bar{p}+D_{1} \rho_{2} \omega^{2} \bar{p},
\end{aligned}
$$

where the function $\bar{p}(\omega, t)$ is defined by

$$
\bar{p}(\omega, t)=\frac{e^{-\lambda(\omega) t} \sinh \left\{t\left[\mu(\omega)^{2}+k\right]^{1 / 2}\right\}}{\left[\mu(\omega)^{2}+k\right]^{1 / 2}} .
$$

On using Sonine's second finite integral as described previously by Lee and Hill [5] we have

$$
\begin{aligned}
\bar{p}(\omega, t) & =\left(\frac{\pi t}{2}\right)^{1 / 2} e^{-\lambda t} \frac{I_{1 / 2}\left[t\left(\mu^{2}+k\right)^{1 / 2}\right]}{\left(\mu^{2}+k\right)^{1 / 4}} \\
& =t e^{-\lambda t} \int_{0}^{\pi / 2} I_{0}\left(k^{1 / 2} t \sin \theta\right) \cosh (\mu t \cos \theta) \sin \theta d \theta \\
& =\frac{e^{-\lambda t}}{2} \int_{-t}^{t} I_{0}\left[k^{1 / 2}\left(t^{2}-\nu^{2}\right)^{1 / 2}\right] e^{\mu \nu} d \nu,
\end{aligned}
$$

and from the Fourier inversion formula,

$$
p(x, t)=\frac{1}{2 \pi} \int_{-\infty}^{\infty} e^{i \omega x} \bar{p}(\omega, t) d \omega=\frac{1}{\pi} \int_{0}^{\infty} \cos x \omega \bar{p}(\omega, t) d \omega,
$$

and the integral,

$$
\int_{0}^{\infty} e^{-\alpha \omega^{2}} \cos x \omega d \omega=\frac{1}{2}\left(\frac{\pi}{\alpha}\right)^{1 / 2} e^{-x^{2} /(4 \alpha)} \quad(\alpha>0),
$$

we may readily deduce

$$
\begin{aligned}
p(x, t) & =\frac{e^{-\left(\gamma_{1}+\gamma_{2}\right) t / 2}}{4 \pi^{1 / 2}} \int_{-t}^{t} e^{\left(\gamma_{1}-\gamma_{2}\right) \nu / 2} \frac{I_{0}\left[k^{1 / 2}\left(t^{2}-\nu^{2}\right)^{1 / 2}\right] e^{-x^{2} /\left(2\left[\left(D_{1}+D_{2}\right) t-\left(D_{1}-D_{2}\right) \nu\right]\right)}}{\left[\frac{\left(D_{1}+D_{2}\right) t}{2}-\frac{\left(D_{1}-D_{2}\right) \nu}{2}\right]^{1 / 2}} d \nu \\
& =\frac{e^{\delta t}}{\left(D_{1}-D_{2}\right)} \int_{D_{2} t}^{D_{1} t} e^{-\gamma \xi} \frac{I_{0}(\eta) e^{-x^{2} /(4 \xi)}}{2(\pi \xi)^{1 / 2}} d \xi,
\end{aligned}
$$


where the final expression follows from the change of variable

$$
\xi=\frac{1}{2}\left[\left(D_{1}+D_{2}\right) t-\left(D_{1}-D_{2}\right) \nu\right]
$$

and the constants $\gamma, \delta$, and the variable $\eta$ are precisely as defined by (4.15) and (4.16) respectively.

Finally from (5.9) we may deduce

$$
\begin{aligned}
& T_{1}(x, t)=\rho_{1}\left(\frac{\partial p}{\partial t}-D_{2} \frac{\partial^{2} p}{\partial x^{2}}\right)+\left(f_{1} \rho_{2}+f_{2} \rho_{1}\right) p+e_{1} \rho_{2} \frac{\partial p}{\partial x} \\
& T_{2}(x, t)=\rho_{2}\left(\frac{\partial p}{\partial t}-D_{1} \frac{\partial^{2} p}{\partial x^{2}}\right)+\left(f_{1} \rho_{2}+f_{2} \rho_{1}\right) p+e_{2} \rho_{1} \frac{\partial p}{\partial x}
\end{aligned}
$$

which together with (5.14) eventually simplify to give

$$
\begin{aligned}
T_{1}(x, t)= & \rho_{1}\left\{\frac{e^{-\gamma_{1} t} e^{-x^{2} /\left(4 D_{1} t\right)}}{2\left(\pi D_{1} t\right)^{1 / 2}}+\frac{k^{1 / 2} e^{\delta t}}{\left(D_{1}-D_{2}\right)} \int_{D_{2} t}^{D_{1} t} e^{-\gamma \xi}\left(\frac{\xi-D_{2} t}{D_{1} t-\xi}\right)^{1 / 2} \frac{I_{1}(\eta) e^{-x^{2} /(4 \xi)}}{2(\pi \xi)^{1 / 2}} d \xi\right. \\
& \left.-\frac{e_{1} e_{2} e^{\delta t}}{\left(D_{1}-D_{2}\right)^{2}} \int_{D_{2} t}^{D_{1} t} e^{-\gamma \xi} \frac{I_{0}(\eta) e^{-x^{2} /(4 \xi)}}{2(\pi \xi)^{1 / 2}} d \xi\right\} \\
& +\rho_{2}\left\{\frac{e^{\delta t}}{\left(D_{1}-D_{2}\right)} \int_{D_{2} t}^{D_{1} t} e^{-\gamma \xi} \frac{I_{0}(\eta) e^{-x^{2} /(4 \xi)}}{2(\pi \xi)^{1 / 2}}\left(f_{1}-\frac{e_{1} x}{2 \xi}\right) d \xi\right\}, \\
T_{2}(x, t)= & \rho_{2}\left\{\frac{e^{-\gamma_{2} t} e^{-x^{2} /\left(4 D_{2} t\right)}}{2\left(\pi D_{2} t\right)^{1 / 2}}+\frac{k^{1 / 2} e^{\delta t}}{\left(D_{1}-D_{2}\right)} \int_{D_{2} t}^{D_{1} t} e^{-\gamma \xi}\left(\frac{D_{1} t-\xi}{\xi-D_{2} t}\right)^{1 / 2} \frac{I_{1}(\eta) e^{-x^{2} /(4 \xi)}}{2(\pi \xi)^{1 / 2}} d \xi\right. \\
& \left.+\frac{e_{1} e_{2} e^{\delta t}}{\left(D_{1}-D_{2}\right)^{2}} \int_{D_{2} t}^{D_{1} t} e^{-\gamma \xi} \frac{I_{0}(\eta) e^{-x^{2} /(4 \xi)}}{2(\pi \xi)^{1 / 2}} d \xi\right\} \\
& +\rho_{1}\left\{\frac{e^{\delta t}}{\left(D_{1}-D_{2}\right)} \int_{D_{2} t}^{D_{1} t} e^{-\gamma \xi} \frac{I_{0}(\eta) e^{-x^{2} /(4 \xi)}}{2(\pi \xi)^{1 / 2}}\left(f_{2}-\frac{e_{2} x}{2 \xi}\right)\right\} .
\end{aligned}
$$

We note that in the above derivation we have made frequent use of the relations

$$
\delta-\gamma D_{j}=-\gamma_{j} \quad(j=1,2)
$$

6. General solutions. Motivated by the structure of the source solutions (5.17) and replacing $\rho_{j} e^{-x^{2} /(4 \xi)} /\left(2(\pi \xi)^{1 / 2}\right)$ by $h_{j}(x, t)(j=1,2)$ where $h_{j}(x, t)$ are assumed to be any solutions of the classical heat equation (2.5), we are led to propose the following 
general solutions of the coupled system (1.2), namely

$$
\begin{aligned}
& T_{1}(x, t)=e^{-\gamma_{1} t} h_{1}\left(x, D_{1} t\right) \\
& +\frac{\gamma_{1}^{1 / 2} e^{\delta t}}{\left(D_{1}-D_{2}\right)} \int_{D_{2} t}^{D_{1} t} e^{-\gamma \xi}\left\{\gamma_{2}^{1 / 2}\left(\frac{\xi-D_{2} t}{D_{1} t-\xi}\right)^{1 / 2} I_{1}(\eta) h_{1}(x, \xi)+\gamma_{1}^{1 / 2} I_{0}(\eta) h_{2}(x, \xi)\right\} d \xi \\
& +\frac{e_{1} e^{\delta t}}{\left(D_{1}-D_{2}\right)} \int_{D_{2} t}^{D_{1} t} e^{-\gamma \xi} I_{0}(\eta)\left\{\frac{\partial h_{2}(x, \xi)}{\partial x}-\frac{e_{2}}{\left(D_{1}-D_{2}\right)}\left[h_{1}(x, \xi)-h_{2}(x, \xi)\right]\right\} d \xi, \\
& T_{2}(x, t)=e^{-\gamma_{2} t} h_{2}\left(x, D_{2} t\right) \\
& +\frac{\gamma_{2}^{1 / 2} e^{\delta t}}{\left(D_{1}-D_{2}\right)} \int_{D_{2} t}^{D_{1} t} e^{-\gamma \xi}\left\{\gamma_{1}^{1 / 2}\left(\frac{D_{1} t-\xi}{\xi-D_{2} t}\right)^{1 / 2} I_{1}(\eta) h_{2}(x, \xi)+\gamma_{2}^{1 / 2} I_{0}(\eta) h_{1}(x, \xi)\right\} d \xi \\
& +\frac{e_{2} e^{\delta t}}{\left(D_{1}-D_{2}\right)} \int_{D_{2} t}^{D_{1} t} e^{-\gamma \xi} I_{0}(\eta)\left\{\frac{\partial h_{1}(x, \xi)}{\partial x}-\frac{e_{1}}{\left(D_{1}-D_{2}\right)}\left[h_{1}(x, \xi)-h_{2}(x, \xi)\right]\right\} d \xi .
\end{aligned}
$$

In order to prove that $(6.1)$ is indeed a formal solution of (1.2) we proceed as follows.

We first decompose (6.1) in the following way:

$$
T_{1}(x, t)=u_{1}(x, t)+v_{1}(x, t), \quad T_{2}(x, t)=u_{2}(x, t)+v_{2}(x, t),
$$

where $\left\{u_{1}, u_{2}\right\}$ are the terms satisfying either (4.13) or (4.14) and $\left\{v_{1}, v_{2}\right\}$ are given by

$$
\begin{aligned}
& v_{1}(x, t)=\frac{e_{1} e^{\delta t}}{\left(D_{1}-D_{2}\right)} \int_{D_{2} t}^{D_{1} t} e^{-\gamma \xi} I_{0}(\eta) \phi_{2}(x, \xi) d \xi, \\
& v_{2}(x, t)=\frac{e_{2} e^{\delta t}}{\left(D_{1}-D_{2}\right)} \int_{D_{2} t}^{D_{1} t} e^{-\gamma \xi} I_{0}(\eta) \phi_{1}(x, \xi) d \xi,
\end{aligned}
$$

where we have introduced $\phi_{j}(x, \xi)(j=1,2)$ such that

$$
\begin{aligned}
& \phi_{1}(x, \xi)=\frac{\partial h_{1}(x, \xi)}{\partial x}-\frac{e_{1}}{\left(D_{1}-D_{2}\right)}\left[h_{1}(x, \xi)-h_{2}(x, \xi)\right], \\
& \phi_{2}(x, \xi)=\frac{\partial h_{2}(x, \xi)}{\partial x}-\frac{e_{2}}{\left(D_{1}-D_{2}\right)}\left[h_{1}(x, \xi)-h_{2}(x, \xi)\right] .
\end{aligned}
$$

We now observe that in terms of the operators $L_{j}^{*}(j=1,2)$ defined by $(4.11)$ the coupled system (1.2) becomes

$$
\begin{aligned}
& L_{1}^{*} T_{1}=\gamma_{1} T_{2}+e_{1}\left\{\frac{\partial T_{2}}{\partial x}-\frac{e_{2}}{\left(D_{1}-D_{2}\right)}\left(T_{1}-T_{2}\right)\right\}, \\
& L_{2}^{*} T_{2}=\gamma_{2} T_{1}+e_{2}\left\{\frac{\partial T_{1}}{\partial x}-\frac{e_{1}}{\left(D_{1}-D_{2}\right)}\left(T_{1}-T_{2}\right)\right\} .
\end{aligned}
$$

Further, if $J(x, t)$ denotes any integral of the form

$$
J(x, t)=\frac{e^{\delta t}}{\left(D_{1}-D_{2}\right)} \int_{D_{2} t}^{D_{1} t} e^{-\gamma \xi} I_{0}(\eta) h(x, \xi) d \xi,
$$

where $h(x, \xi)$ is a solution of the classical heat equation (2.5) then, by applying the substitution

$$
\xi=D_{1} t-\left(D_{1}-D_{2}\right) \tau,
$$


we may deduce, by partial differentiation with respect to time, that

$$
L_{1}^{*} J=e^{-\gamma_{2} t} h\left(x, D_{2} t\right)+\frac{k^{1 / 2} e^{\delta t}}{\left(D_{1}-D_{2}\right)} \int_{D_{2} t}^{D_{1} t} e^{-\gamma \xi}\left(\frac{D_{1} t-\xi}{\xi-D_{2} t}\right)^{1 / 2} I_{1}(\eta) h(x, \xi) d \xi,
$$

while similarly the substitution,

$$
\xi=D_{2} t+\left(D_{1}-D_{2}\right) \tau,
$$

yields

$$
L_{2}^{*} J=e^{-\gamma_{1} t} h\left(x, D_{1} t\right)+\frac{k^{1 / 2} e^{\delta t}}{\left(D_{1}-D_{2}\right)} \int_{D_{2} t}^{D_{1} t} e^{-\gamma \xi}\left(\frac{\xi-D_{2} t}{D_{1} t-\xi}\right)^{1 / 2} I_{1}(\eta) h(x, \xi) d \xi .
$$

Finally, by direct calculation from (6.1), we may establish

$$
\begin{aligned}
& e_{2}\left\{\frac{\partial T_{1}}{\partial x}-\frac{e_{1}}{\left(D_{1}-D_{2}\right)}\left(T_{1}-T_{2}\right)\right\} \\
&= e_{2}\left\{e^{-\gamma_{1} t} \phi_{1}\left(x, D_{1} t\right)+\frac{k^{1 / 2} e^{\delta t}}{\left(D_{1}-D_{2}\right)} \int_{D_{2} t}^{D_{1} t} e^{-\gamma \xi}\left(\frac{\xi-D_{2} t}{D_{1} t-\xi}\right)^{1 / 2} I_{1}(\eta) \phi_{1}(x, \xi) d \xi\right\} \\
& \quad-\frac{e_{1} \gamma_{2} e^{\delta t}}{\left(D_{1}-D_{2}\right)} \int_{D_{2} t}^{D_{1} t} e^{-\gamma \xi} I_{0}(\eta) \phi_{2}(x, \xi) d \xi, \\
& e_{1}\left\{\frac{\partial T_{2}}{\partial x}-\frac{e_{2}}{\left(D_{1}-D_{2}\right)}\left(T_{1}-T_{2}\right)\right\} \\
&= e_{1}\left\{e^{-\gamma_{2} t} \phi_{2}\left(x, D_{2} t\right)+\frac{k^{1 / 2} e^{\delta t}}{\left(D_{1}-D_{2}\right)} \int_{D_{2} t}^{D_{1} t} e^{-\gamma \xi}\left(\frac{D_{1} t-\xi}{\xi-D_{2} t}\right)^{1 / 2} I_{1}(\eta) \phi_{2}(x, \xi) d \xi\right\} \\
&-\frac{e_{2} \gamma_{1} e^{\delta t}}{\left(D_{1}-D_{2}\right)} \int_{D_{2} t}^{D_{1} t} e^{-\gamma \xi} I_{0}(\eta) \phi_{1}(x, \xi) d \xi .
\end{aligned}
$$

On substituting (6.2) into (6.5) and exploiting the above equations and (4.13) we may readily confirm that $(6.1)$ constitutes a formal solution of (1.2) for arbitrary heat functions $h_{j}(x, t)(j=1,2)$. We emphasize that in verifying this solution we have made use of the fundamental relation $(1.4)_{2}$ between the constants $e_{j}$ and $f_{j}$ $(j=1,2)$. Unfortunately, because the general solutions $(6.1)$ also involve $\partial h_{j} / \partial x$ as well as $h_{j}(j=1,2)$ this result is not as useful in the solution of boundary value problems as the expressions (2.2). Accordingly we illustrate these results for the case of equal heat capacities and we consider a special case of the problem considered in some detail by Clarke [1].

7. Simple illustrative example. In the special case of equal heat capacities $\nu_{1}=$ $\nu_{2}=\nu$, the flux equations (3.5) have solutions of the form (2.2)-(2.4) with $F_{j}$ in place of $f_{j}(j=1,2)$ and the constants are given by

$$
\begin{array}{lll}
D_{1}=\frac{\lambda_{1}}{\nu}, & E_{1}=0, & F_{1}=\beta^{2} p_{2} \frac{\lambda_{1}}{\nu}, \\
D_{2}=\frac{\lambda_{2}}{\nu}, & E_{2}=0, & F_{2}=\beta^{2} p_{1} \frac{\lambda_{2}}{\nu} .
\end{array}
$$


For the problem of the semi-infinite medium $0<x<\infty$ with constant flux $Q_{0}$ on the plane $x=0$ and zero temperature at infinity, the appropriate boundary conditions become, in view of $(3.1)$,

$$
Q_{j}(0, t)=Q_{0}, \quad Q_{j}(\infty, t)=0 \quad(j=1,2) .
$$

Now, as described in Hill [3], such conditions can be realized by expressions of the form (2.2) for $Q_{1}(x, t)$ and $Q_{2}(x, t)$ provided that $h_{j}(x, t)(j=1,2)$ satisfy the same boundary data, namely,

$$
h_{j}(0, t)=Q_{0}, \quad h_{j}(\infty, t)=0 \quad(j=1,2) .
$$

Assuming zero initial condition the appropriate heat functions are simply

$$
h_{j}(x, t)=Q_{0} \operatorname{erfc}\left(\frac{x}{2 t^{1 / 2}}\right) \quad(j=1,2) .
$$

Thus, altogether, the solution of the flux equations (3.5) in the special case $\nu_{1}=$ $\nu_{2}=\nu$ becomes

$$
\begin{aligned}
& \frac{Q_{1}(x, t)}{Q_{0}}=e^{-F_{1} t} \operatorname{erfc}\left(\frac{x}{2\left(D_{1} t\right)^{1 / 2}}\right) \\
& \quad+\frac{F_{1}^{1 / 2} e^{\bar{\delta} t}}{\left(D_{1}-D_{2}\right)} \int_{D_{2} t}^{D_{1} t} e^{-\bar{\eta} \xi}\left\{F_{2}^{1 / 2}\left(\frac{\xi-D_{2} t}{D_{1} t-\xi}\right)^{1 / 2} I_{1}(\bar{\eta})+F_{1}^{1 / 2} I_{0}(\bar{\eta})\right\} \operatorname{erfc}\left(\frac{x}{2 \xi^{1 / 2}}\right) d \xi, \\
& \frac{Q_{2}(x, t)}{Q_{0}}=e^{-F_{2} t} \operatorname{erfc}\left(\frac{x}{2\left(D_{2} t\right)^{1 / 2}}\right) \\
& \quad+\frac{F_{2}^{1 / 2} e^{\bar{\delta} t}}{\left(D_{1}-D_{2}\right)} \int_{D_{2} t}^{D_{1} t} e^{-\bar{\eta} \xi}\left\{F_{1}^{1 / 2}\left(\frac{D_{1} t-\xi}{\xi-D_{2} t}\right)^{1 / 2} I_{1}(\bar{\eta})+F_{2}^{1 / 2} I_{0}(\bar{\eta})\right\} \operatorname{erfc}\left(\frac{x}{2 \xi^{1 / 2}}\right) d \xi,
\end{aligned}
$$

where $\bar{\gamma}, \bar{\delta}$, and $\bar{\eta}$ are given by (2.3) and (2.4) but with $F_{j}$ in place of $f_{j}(j=1,2)$. Finally, the expected conditional temperatures may then be deduced from the above and equation (3.7).

Acknowledgment. The financial support of one of us (J. M. Hill) from the Science and Engineering Research Council is gratefully acknowledged.

\section{REFERENCES}

[1] N. S. Clarke, Heat diffusion in random laminates, Quart. J. Mech. Appl. Math. 37, 195-230 (1984)

[2] N. S. Clarke, On the effective diffusivity model for heat diffusion in inclined random laminates, Quart. J. Mech. Appl. Math. 39, 139-152 (1986)

[3] J. M. Hill, On the solution of reaction-diffusion equations, IMA J. Appl. Math. 27, 177-194 (1981)

[4] A. I. Lee and J. M. Hill, On the solution of boundary value problems for fourth order diffusion, Acta Mech. 46, 23-35 (1983)

[5] A. I. Lee and J. M. Hill, On the general linear coupled system for diffusion in media with two diffusivities, J. Math. Anal. Appl. 89, 530-557 (1982)

[6] J. R. Willis and J. M. Hill, On heat diffusion in random laminates, Quart. J. Mech. Appl. Math. 41 (1988) 